\title{
TREINOS DISCRIMINATIVOS EM CÃES UTILIZANDO ESTÍMULOS VISUAIS E PROCEDIMENTO AUTOMATIZADO
}

DISCRIMINATIVE TRAINING IN DOGS USING VISUAL STIMULI AND AUTOMATED PROCEDURE

\author{
EDSON MASSAYUKI HUZIWARA \\ UNIVERSIDADE FEDERAL DE MINAS GERAIS E \\ INSTITUTO NACIONAL DE CIÊNCIA E TECNOLOGIA SOBRE COMPORTAMENTO COGNIÇÃO E ENSINO, BRASIL
}

\author{
TATHIANNA AMORIM SOUZA MONTAGNOLI \\ UNIVERSIDADE FEDERAL DE SÃo CARLOS, BRASIL \\ DEISY DAS GRAÇAS DE SOUZA \\ UNIVERSIDADE FEDERAL DE SÃo CARLOS, BRASIL E \\ InSTITUTO NACIONAL DE CIÊNCIA E TECNOLOGIA SOBRE COMPORTAMENTO COGNIÇÃO E ENSINO, BRASIL
}

\section{RESUMO}

Esse artigo apresenta dois experimentos que tiveram por objetivo estabelecer discriminações condicionais visualvisuais em cães utilizando um aparato automatizado. No Experimento I, conduzido com três cães, procurou-se estabelecer discriminações condicionais utilizando um procedimento em que cada relação modelo-comparação era apresentada em blocos de tentativas distintos. No Experimento II, conduzido com dois cães, nós utilizamos uma adaptação de um protocolo de ensino de repertórios discriminativos (partindo de discriminações simples até discriminações condicionais) para pessoas com necessidades educacionais especiais. Em ambos os experimentos, os desempenhos dos sujeitos ficaram sob controle de discriminações simples ao invés de discriminações condicionais. Ao considerar que cães geralmente aprendem discriminações condicionais auditivo-visuais, esses resultados parecem representar um desafio para análises atuais e, ao mesmo tempo, parecem dar origens a dúvidas que poderão ser experimentalmente investigadas no futuro.

Palavras-chave: discriminação simples, discriminação condicional, estímulos visuais, aparato automatizado, cães.

\section{ABSTRACT}

This paper presents two experiments that aimed to establish visual-visual conditional discriminations in dogs using an automated device. In Experiment I, conducted with 3 dogs, we tried to establish conditional discriminations using a procedure in which each sample-comparison relation was presented in one separated block of trials. In Experiment II, carried out with two dogs, we used an adapted version of a training path to establish discriminative repertoires (from simple to conditional discriminations) in people with special educational needs. In both experiments, subjects' performances were controlled by simple discriminations instead of conditional discriminations. Considering that dogs usually learn auditory-visual conditional discriminations, these results seem to represent a challenge for current analysis and, at same time, seem to give rise to doubts that could be experimentally investigated in the future.

Keywords: simple discriminations, conditional discrimination, visual stimuli, automated device, dogs.

O presente trabalho deriva de pesquisa financiada pelo CNPq (Processo \#573972/2008-7) e pela FAPESP (Processo \#2008/57705-8) no âmbito do Instituto Nacional de Ciência e Tecnologia sobre Comportamento, Cognição e Ensino (INCT-ECCE). Correspondência relativa ao presente artigo deve ser enviada ao primeiro autor: huziwara.edson@gmail.com 
Estudos recentes têm investigado as habilidades comportamentais de cães domésticos em diversos contextos (para revisão completa, ver Udell \& Wynne, 2008). Alguns desses experimentos descreveram, por exemplo, efeitos de dicas sociais e gestos apresentados por pessoas sobre o comportamento de escolha dos animais (Dorey, Udell, \& Wynne, 2009; Hare, Call, \& Tomazello, 1998; Ittyerah \& Gaunet, 2009). Outros estudos investigaram as estratégias adotadas e os aspectos ambientais que controlam o comportamento de cães em contextos de procura por objetos escondidos (Fiset, Landry, \& Ouellette, 2006; Gazit, Goldblatt, \& Terkel, 2005).

No que diz respeito a habilidades cognitivas, encontram-se experimentos que avaliaram a capacidade dos cães em utilizar símbolos arbitrários na comunicação com humanos (Rossi \& Ades, 2008) ou integrar informações auditivas e visuais (Adachi, Kuwahata, \& Fujita, 2007). A aprendizagem por imitação (Heberlein \& Turner, 2009; Topál, Byrne, Miklósi, \& Csányi, 2006), o conceito piagetiano de permanência do objeto (Fiset \& LeBlanc, 2007; Miller, Rayburn-Reeves, \& Zentall, 2009) e estratégias de resolução de problemas (Osthaus, Lea, \& Slater, 2005) também foram estudados com esses sujeitos.

O experimento de Kaminski, Call e Fischer (2004), por exemplo, foi conduzido com um cão border collie chamado Rico, o qual apresentava cerca de 200 relações condicionais auditivo-visuais, ou seja, sob controle do comando verbal específico, esse cão era capaz de selecionar corretamente cada um desses 200 itens. No referido experimento, a partir desta linha de base de relações condicionais auditivo-visuais, Kaminsk et al. avaliaram o responder por exclusão (Dixon, 1977) na identificação de novos itens. Nas sessões experimentais, enquanto Rico e seu dono esperavam em uma sala adjacente, o pesquisador dispunha oito objetos na sala experimental. Destes, sete objetos pertenciam à linha de base e um deles era completamente novo. $\mathrm{O}$ experimentador instruía o dono do cão a requisitar verbalmente um dos itens disponíveis na sala experimental. A tarefa de Rico consistia em dirigir-se à sala experimental e trazer o objeto correspondente ao nome dito pelo dono. $\mathrm{Na}$ primeira oportunidade de resposta, o experimentador sempre requisitava que Rico trouxesse um objeto que pertencia à linha de base. Em uma tentativa subsequente, geralmente na segunda ou terceira oportunidades de resposta, o experimentador instruía o dono a pronunciar um nome desconhecido. A resposta seria considerada correta caso Rico excluísse os objetos conhecidos e escolhesse o único objeto novo do conjunto apresentado. O procedimento introduzia um objeto novo por sessão e foram conduzidas dez sessões, nas quais tanto os objetos familiares quanto os novos eram trocados. Rico escolheu o objeto novo diante do nome desconhecido em sete das dez oportunidades de resposta, caracterizando assim tanto o responder controlado por relações condicionais auditivo-visuais (nome-objeto), quanto o responder controlado por exclusão dos itens envolvidos em outras relações condicionais.
Um segundo exemplo de responder por exclusão foi apresentado em um conjunto de experimentos conduzidos por Pilley e Reid (2011) e que utilizaram uma cachorra de nome Chaser, também da raça border collie. Os dois primeiros experimentos relataram os treinos e os testes que foram realizados durante um período de três anos para comprovar que Chaser era capaz de adquirir e manter relações condicionais auditivo-visuais para, aproximadamente, 1.000 itens. O terceiro experimento demonstrou que Chaser foi capaz de aprender relações de palavras que representavam categorias, ou seja, situações nas quais uma mesma palavra estaria relacionada a diversos objetos e, também, situações nas quais um mesmo objeto poderia receber diferentes nomes. $\mathrm{O}$ Experimento 4, por fim, demonstrou a capacidade de Chaser em responder por exclusão, similar a habilidade que fora demonstrada com Rico (Kaminsk et al., 2004).

Um terceiro exemplo de experimento que investigou as habilidades cognitivas em cães foi conduzido por Range, Aust, Steurer e Huber (2008) e contou com a utilização de um aparato automatizado para apresentação dos estímulos, registro das respostas e liberação do reforçador. A utilização de tais aparatos previne que dicas sociais espúrias emitidas pelos experimentadores venham a controlar o comportamento dos cães e interferir nos resultados obtidos (Montagnoli, 2012). Range et al. ensinaram discriminações simples utilizando 80 fotografias divididas em dois conjuntos e apresentadas em uma tela sensível ao toque. O Conjunto A era composto por 40 diferentes fotografias de cães; o Conjunto B era composto por 40 diferentes fotografias de paisagens (sem cães). Cada tentativa era iniciada com a apresentação simultânea de duas fotografias na tela, uma que pertencia ao Conjunto A e outra pertencente ao Conjunto B. Todas as configurações de pares entre as fotografias dos conjuntos A e B foram utilizadas. Tocar com o focinho em uma das fotografias encerrava a tentativa. Respostas aos estímulos do Conjunto A produziam o acionamento do dispensador de comida. Respostas aos estímulos do conjunto B produziam um período de $3 \mathrm{~s}$ em que a tela ficava vermelha, seguido pela reapresentação da mesma tentativa. Uma sessão experimental tinha 30 tentativas e o critério de aprendizagem era a obtenção de escores iguais ou superiores a $80 \%$ de acertos em quatro de cinco sessões consecutivas. Todos os quatro sujeitos submetidos ao procedimento atingiram o critério de aprendizagem, atestando assim a possibilidade de se utilizar estímulos visuais apresentados em telas sensíveis ao toque para o ensino de discriminações simples para cães.

Udell e Wynne (2008) consideraram que a produção substancial e sistemática de pesquisas sobre cães domesticados nos últimos 20 anos se deve ao fato de que, em determinadas tarefas, esses sujeitos apresentam desempenhos superiores aos descritos em primatas não humanos, tradicionalmente utilizados como modelos animais para a comparação com o comportamento humano. Em função disso, estudos conduzidos com cães podem ser úteis para a compreensão de processos comportamentais básicos, possivelmente compartilhados com humanos, e que podem estar na origem da aquisição 
de comportamento simbólico (Montagnoli, 2012; Rossi \& Ades, 2008).

O presente artigo descreve dois experimentos que buscaram verificar o potencial de um aparato automatizado que requer a resposta operante de focinhar uma tela sensível ao toque em procedimentos para estabelecer relações condicionais visual-visuais em cães. Esta é uma habilidade comportamental ainda pouco descrita com esses sujeitos (Callahan, Ikeda-Douglas, Head, Cotman, \& Milgram, 2000; Kaminski, Tempelmann, Call, \& Tomasello, 2009) e a utilização de um aparato como o descrito por Range et al. (2008) possibilitaria a avaliação de propriedades relacionais emergentes (i.e., não diretamente ensinadas), tais como simetria ou emparelhamento por identidade generalizada. Resultados positivos poderiam recomendar o uso do equipamento e indicar o procedimento como um passo inicial para um programa de estudos sobre a formação de classes de estímulos equivalentes e comportamento simbólico (Sidman \& Tailby, 1982) em cães. De forma mais específica, no Experimento I, conduzido com três cães, procurou-se estabelecer discriminações condicionais utilizando um procedimento em que cada relação modelocomparação era apresentada em blocos de tentativas distintos. No Experimento II, conduzido com dois cães, utilizou-se uma adaptação de um protocolo de ensino de repertórios discriminativos (partindo de discriminações simples até discriminações condicionais) para pessoas com necessidades educacionais especiais.

\section{EXPERIMENTO I MÉTODO}

\section{Sujeitos}

Participaram dos experimentos dois cães domésticos (Canis familiaris), um macho e uma fêmea. Todos os cães eram experimentalmente ingênuos e residiam em clínicas veterinárias particulares. A Tabela 1 apresenta as principais características de cada um dos sujeitos.

\section{Local e situação experimental}

A coleta era realizada cinco vezes por semana, na própria clínica em que viviam os animais. Nos locais disponíveis para coleta, que mediam $3 \times 3 \mathrm{~m}$ aproximadamente, eram dispostos o aparato de coleta e um microcomputador portátil. Os cães não eram submetidos a qualquer forma de restrição física e, por esse motivo, foi necessário modelar a resposta de permanecer diante da tela e focinhar nos estímulos apresentados durante a sessão (ver descrição a seguir).

Tabela 1. Características dos sujeitos: Nome, idade aproximada, gênero, raça e os experimentos aos quais foram submetidos.

\begin{tabular}{ccccc}
\hline Nome & $\begin{array}{c}\text { Idade } \\
\text { Aproximada }\end{array}$ & $\begin{array}{c}\text { Gêner } \\
\text { o }\end{array}$ & Raça & Experimento \\
\hline Furacão & 03 anos & $\begin{array}{c}\text { Mach } \\
\text { o }\end{array}$ & Indefinido & I \\
Norma & 04 anos & Fêmea & Indefinido & I e II \\
Pipoca & 01 ano & Fêmea & Schnauzer & II \\
\hline
\end{tabular}

Para aproveitar a condição natural de privação alimentar, as sessões eram conduzidas antes de os sujeitos receberem a primeira refeição do dia, entre $8 \mathrm{~h}$ e $09 \mathrm{~h} 30 \mathrm{~min}$ da manhã. As sessões eram realizadas individualmente e tinham duração média de $15 \mathrm{~min}$. Após as sessões, os cães eram alimentados pelos experimentadores.

\section{Equipamento}

Para a coleta de dados, foi construído um aparato de madeira de 40x70x40cm (largura, altura, comprimento). Na parede frontal do aparato foi acoplada uma tela sensível ao toque Elo Touch Systems ${ }^{\circledR}$ de 15 ", no qual os sujeitos deveriam emitir as respostas de focinhar. A altura de apresentação da tela era regulada de acordo com a altura de cada sujeito. Abaixo da tela e $3 \mathrm{~cm}$ acima do solo era alocada uma abertura retangular utilizada para administrar a entrega dos estímulos reforçadores. A unidade de ração utilizada como estímulo reforçador era dispensada pelo experimentador, por meio de um tubo de PVC de $20 \mathrm{~cm}$. A ração percorria o tubo e caia em um recipiente plástico localizado abaixo do monitor. Como o tubo ficava atrás do monitor, o manuseio e liberação da unidade de ração não podiam ser vistos pelo animal durante o experimento (ver Figura 1).

A apresentação de estímulos, o gerenciamento dos procedimentos de ensino e o registro de dados eram realizados por um microcomputador portátil equipado com um software desenvolvido especificamente para conduzir experimentos com sujeitos não humanos (Velasco \& Picorone, 2008). O software também era o responsável por liberar consequências sonoras diferenciais para acertos e erros por meio de duas caixas de som próprias para computador alocadas no chão. Um padrão sonoro agudo, semelhante a uma campainha residencial, com duração de $3 \mathrm{~s}$ foi correlacionado com respostas corretas durante todo o procedimento. Um padrão sonoro grave, semelhante ao som de uma buzina, também com duração de $3 \mathrm{~s}$ foi correlacionado com respostas incorretas.
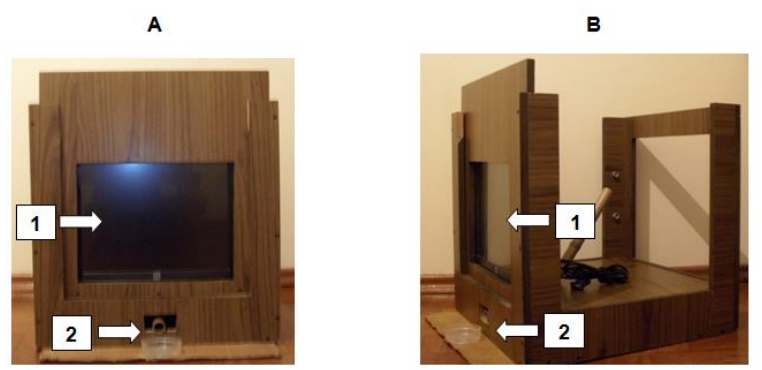

Figura 1. Vista frontal (Painel A) e lateral do aparato (Painel B). Em ambos os painéis são apresentados (1) a tela do computador sensível ao toque; e (2) a saída do dispensador e o recipiente plástico no qual a ração canina era coletada.

\section{Estímulos Visuais}

Os estímulos visuais eram figuras abstratas desenhadas em preto sobre um fundo branco que mediam $3 \times 3 \mathrm{~cm}$ (ver Tabela 2). Dispostos horizontalmente, os estímulos eram apresentados na tela em três diferentes posições (esquerda, central e direita), mantendo entre si uma distância de $6 \mathrm{~cm}$, medidos de centro a centro de cada estímulo. 
Tabela 2. Estímulos visuais utilizados no Pré-treino (X) e na fase de ensino de relações de identidade nos Experimentos I (A e B) e II (C e D).

\begin{tabular}{cccccc}
\hline & $\mathrm{X}$ & $\mathrm{A}$ & $\mathrm{B}$ & $\mathrm{C}$ & $\mathrm{D}$ \\
\hline 1 & 10 & & & 0 & 0
\end{tabular}

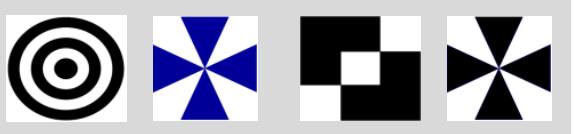

Procedimento

Contingências programadas no decorrer do experimento

As tentativas eram iniciadas com a apresentação de um estímulo modelo na posição central da tela. Focinhar esse estímulo produzia a retirada do modelo e a apresentação imediata de dois estímulos de comparação nas posições laterais da tela, sendo um deles fisicamente idêntico ao modelo. Tanto a ordem de apresentação dos modelos quanto a posição de apresentação dos estímulos de comparação eram balanceadas. A escolha do comparação idêntico ao modelo era considerada correta e produzia o padrão sonoro agudo e, simultaneamente, a entrega de uma unidade de ração canina. Em seguida, tinha início um intervalo entre tentativas (IET) de $2 \mathrm{~s}$. Durante o IET, era apresentada uma tela branca e eventuais respostas emitidas pelos sujeitos não produziam alterações ambientais programadas.

Escolher o comparação diferente do modelo era considerado como uma resposta incorreta e produzia simultaneamente a apresentação do padrão sonoro grave, o escurecimento da tela (time out) por $3 \mathrm{~s}$ e o IET, além da reapresentação da mesma tentativa. Após o terceiro erro consecutivo na mesma tentativa, apenas o comparação correto era apresentado na próxima oportunidade de resposta (quarta tentativa), manipulação que forçava o sujeito a focinhar nessa comparação (Procedimento de correção A).

A utilização do Procedimento de correção B foi necessária uma vez que apenas as consequências diferenciais para acertos e erros não foram suficientes para gerar o aprendizado esperado no caso de Norma. O Procedimento de correção B consistia em apresentar 10 sessões consecutivas em que respostas ao estímulo de comparação incorreto eram colocadas em extinção. Por exemplo, em uma tentativa na qual o estímulo A1 era apresentado como modelo, os estímulos de comparação seriam A1 e A2. O sujeito poderia focinhar inúmeras vezes o comparação $\mathrm{A} 2$, mas essa resposta não produzia alterações no ambiente. A tentativa era encerrada se, e somente se, o sujeito focinhasse o comparação A1. Após aplicação desse conjunto de 10 sessões, a escolha do estímulo de comparação incorreto era novamente liberada e produzia as consequências anteriormente descritas para erros (Procedimento de correção A).

\section{Modelagem da resposta alvo}

Inicialmente os cães eram treinados a coletar unidades de ração canina dirigindo-se ao comedouro. Nessa etapa, o padrão sonoro agudo era pareado com a entrega da ração. Para fazer um paralelo com o processo de modelagem que ocorre com ratos em caixa de condicionamento operante, o padrão sonoro no presente experimento equivaleria ao som do acionamento do dispensador de comida na caixa de condicionamento operante. Assim sendo, o treino ao comedouro era mantido até que a apresentação do padrão sonoro controlasse fielmente a resposta de se dirigir ao comedouro e coletar a ração, sinalizando que o sujeito havia adquirido o condicionamento entre apresentação do padrão sonoro e a obtenção de comida.

Em seguida, tinha início a modelagem da resposta de focinhar a tela. Durante a modelagem, a tela apresentava o estímulo X1 (ver Tabela 2) na posição central. Na primeira etapa, era reforçada a resposta de olhar para a tela; na segunda etapa, eram reforçadas aproximações à tela; na terceira etapa, a resposta de encostar o focinho em qualquer parte da tela era reforçada. Nessas três primeiras etapas, o software não detectava as respostas emitidas pelos sujeitos e o reforço era administrado segundo os critérios estabelecidos pelos experimentadores. Finalmente, na quarta etapa, a resposta de focinhar a tela sobre o estímulo visual era registrada automaticamente e indicava ao experimentador quando entregar a ração. A exigência para a resposta em cada etapa aumentava apenas quando a resposta da etapa anterior estivesse sendo emitida com alta frequência e de forma regular.

Uma vez estabelecida a resposta alvo, foram conduzidas cinco sessões de fortalecimento dessa resposta. Cada sessão tinha 80 tentativas nas quais o estímulo X1 era apresentado de forma alternada nas três posições a serem utilizadas durante a tarefa experimental.

\section{Ensino de discriminações condicionais}

O ensino de discriminações condicionais, iniciado logo após a modelagem da resposta, utilizava o procedimento do treino blocado (Saunders \& Spradlin, 1990, 1993). Nesse procedimento, cada uma das relações condicionais modelo-comparação é apresentada em blocos distintos de tentativas. Ao longo de sucessivas sessões, a quantidade de tentativas nos blocos foi gradualmente diminuída até o momento em que apresentação do modelo tornou-se aleatória. Em virtude dos resultados obtidos pelos sujeitos (ver seção de Reultados e Discussão), no entanto, optou-se por não realizar a diminuição na quantidade de tentativas por bloco, motivo pelo qual foram conduzidas apenas as Condições 1 e 2 que são descritas a seguir.

Na Condição 1, as sessões tinham 40 tentativas em que apenas o estímulo A1 era apresentado como modelo e os estímulos A1 e A2 eram simultaneamente apresentados como comparações. O critério de aprendizagem consistiu em três de quatro sessões consecutivas com porcentagens iguais ou superiores a $80 \%$ de acertos. Atingido o critério de aprendizagem na Condição 1, os sujeitos iniciaram a Condição 2, também 
com sessões de 40 tentativas. Nesta condição apenas o estímulo A2 era apresentado como modelo, enquanto os estímulos de comparação continuavam a ser A1 e A2. O mesmo critério de aprendizagem foi adotado.

Uma vez atingido o critério de aprendizagem na Condição 2, tinha início a repetição da Condição 1 . Furacão foi submetido a oito reversões entre as Condições 1 e 2, enquanto Norma foi submetida apenas a quatro reversões.

\section{RESULTADOS E DISCUSSÃO}

A Figura 2 apresenta os resultados obtidos por Furacão e Norma nos passos de ensino do Experimento I. Círculos preenchidos representam as porcentagens de acertos nas sessões da Condição 1 e círculos vazios representam as porcentagens de acertos nas sessões da Condição 2. A linha tracejada horizontal identifica a porcentagem mínima de acertos exigida para a obtenção do critério de aprendizagem. Os momentos nos quais o Procedimento de Correção $B$ foi utilizado são identificados pela descontinuidade dos percentuais de acertos. A quantidade de sessões necessária para concluir cada passo é indicada no eixo $\mathrm{X}^{1}$.

$\mathrm{O}$ procedimento de ensino no Experimento I previa a utilização de um treino de relações condicionais em blocos de tentativas (Saunders \& Spradlin, 1990, 1993). Com a finalidade de maximizar as oportunidades de acertos dos sujeitos, optou-se por iniciar o procedimento utilizando condições em que apenas uma relação modelocomparação era apresentada (Condição 1 - relação A1-A1; Condição 2 - relação A2-A2). Esperava-se que, ao longo das sucessivas alternâncias entre as Condições 1 e 2, a quantidade de sessões para a obtenção do critério fosse diminuindo de forma gradual. No momento em que os sujeitos estivessem atingindo o critério para cada uma das Condições em um número reduzido de sessões (i.e., duas ou três), ambas as relações seriam apresentadas em uma mesma sessão, ainda separadas por blocos de tentativas (p. ex., 20 tentativas iniciais apresentando a relação A1-A1 e as 20 tentativas finais apresentando a relação A2-A2). Nas condições seguintes, a quantidade de tentativas nos blocos seria gradualmente diminuída até o momento em que apresentação das relações modelo-comparação tornar-se-ia aleatória. Tal procedimento tinha o objetivo de estabelecer um desempenho controlado por relações condicionais baseadas na similaridade física dos estímulos.

Furacão foi exposto a um total de 106 sessões. As porcentagens de acertos estiveram próximas ao nível do acaso durante as primeiras sessões da primeira exposição à Condição 1. Entretanto, ao longo das sessões, os acertos aumentaram de forma rápida e constante e o critério de acertos para essa condição, que apresentava A1 como modelo, foi obtido após 13 sessões. De forma alternada, Furacão foi exposto ainda a mais três exposições da

\footnotetext{
${ }^{1}$ Nas sessões com o Procedimento de Correção B, respostas ao S- não produziam qualquer alteração ambiental programada e a tentativa era encerrada apenas quando uma resposta $\mathrm{S}+$ fosse emitida e o percentual de acertos foi de $100 \%$ em todos esses casos. Por esse motivo, os dados referentes a tais sessões não foram apresentados na Figura 2 e também foram desconsideradas para calcular a quantidade de sessões necessárias até critério.
}

Condição 1 (relação A1-A1) e quatro exposições da Condição 2 (relação A2-A2). Nessas sessões, o sujeito apresentou um padrão de desempenho recorrente: o índice de acertos foi baixo nas sessões que ocorreram imediatamente após uma reversão, chegando a atingir $0 \%$ de acertos em algumas sessões; ao longo de repetidas exposições a sessões de uma mesma condição, o índice de acertos foi aumentando gradativa e consistentemente e o critério de aprendizagem foi atingido, em média, a partir da $13^{\mathrm{a}}$ sessão. Exceção feita a segunda exposição da Condição 1 , em que o critério foi atingido após a $20^{\mathrm{a}}$ sessão.

Norma foi exposta a 133 sessões (desconsiderando-se aquelas em que foi utilizado o Procedimento de Correção B). O desempenho de Norma não apresentou um padrão ascendente e gradativo como Furacão. Apesar de os índices de acertos também serem baixos nas sessões que ocorreram imediatamente após uma reversão, as oscilações de uma sessão para outra foram muito mais acentuadas que as observadas em Furacão, de forma que o critério de aprendizagem foi atingido em um número muito maior de sessões (em média, foram necessárias 33 sessões por condição no caso de Norma, versus 13 no caso de Furacão).

Ainda sobre o desempenho de Norma, na primeira exposição à Condição 1 , o procedimento de correção B não precisou ser utilizado. No entanto, nos demais passos, a quantidade de ocorrências desse procedimento aumentou gradativamente, sendo necessária uma apresentação do procedimento de correção B na primeira exposição à Condição 2, duas apresentações na segunda exposição à Condição 1 e três apresentações na segunda exposição à Condição 2. A partir desse fato, pode-se supor que as reversões nas funções dos estímulos de comparação (ora A1 era $\mathrm{S}+$ ora era $\mathrm{S}$-) tenham exercido um efeito deletério no desempenho do animal.

A análise dos resultados sugere, portanto, que essa organização do treino originou um desempenho controlado por discriminação simples, ou seja, os sujeitos ficaram sob controle somente do estímulo que sinalizava reforço em cada Condição, independentemente do estímulo modelo que o antecedia. Porcentagens próximas a $0 \%$ de acertos quando ocorria a alternância entre condições, tipicamente observada em procedimentos que utilizam reversões de discriminações simples, parecem confirmar tal análise.

Como dito anteriormente, ao considerar que $(i)$ o procedimento de treino permitia o estabelecimento de discriminações simples e, também, que (ii) ocorria a reversão da discriminação a partir da alternância entre as Condições 1 e 2, é possível comparar os presentes resultados com aqueles obtidos em treinos de discriminações simples com reversões sucessivas (Capaldi \& Senko, 1961; Goulart, Galvão, \& Barros, 2003; Harlow, 1949; Vaughan, 1988). De forma geral, tais experimentos descreveram um padrão comportamental denominado de learning set. Segundo os autores, a ocorrência de learning set é caracterizada por uma relação inversa entre a quantidade de sessões de treino até critério e a quantidade de reversões realizadas. 


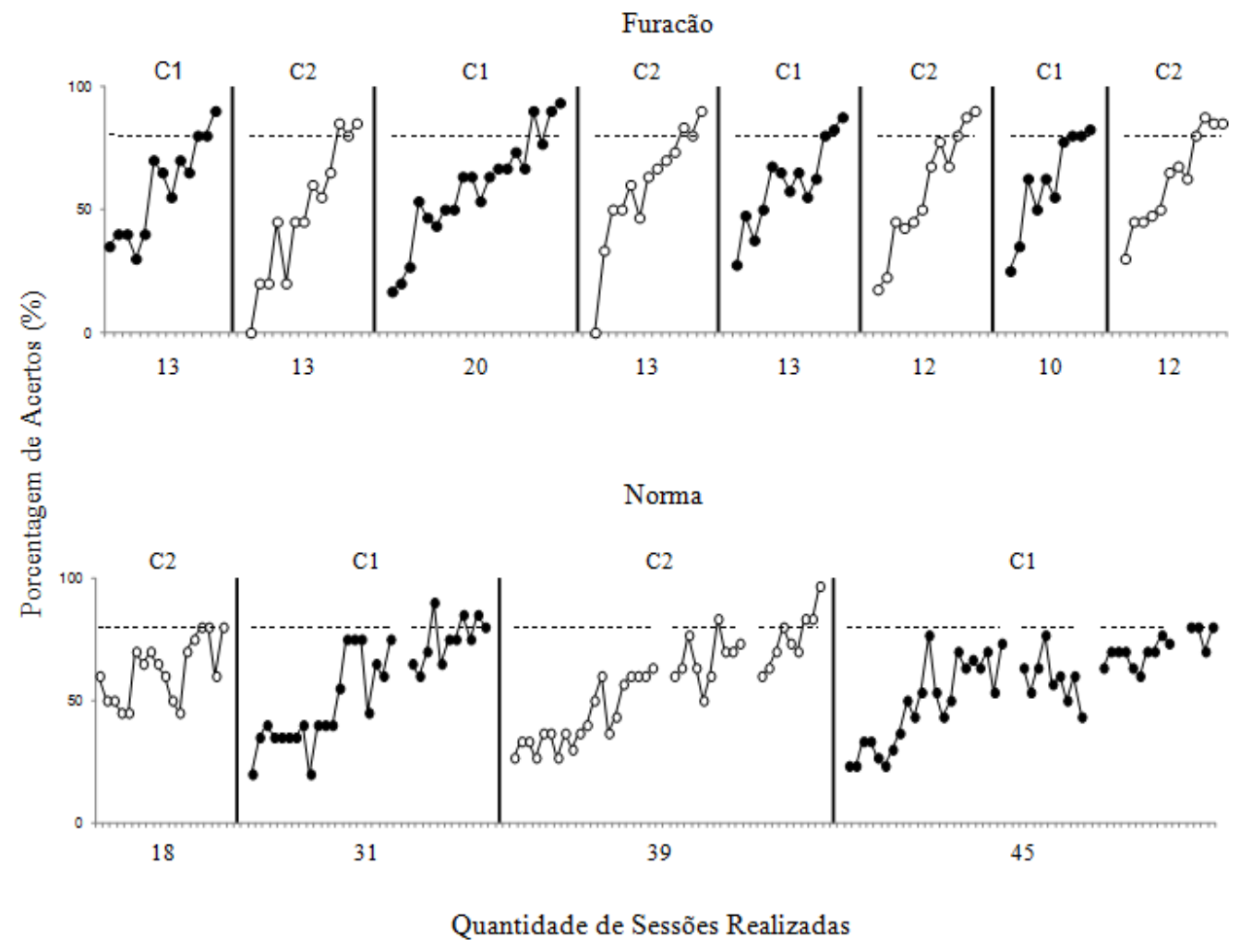

Figura 2. Porcentagens de acertos obtidos pelos sujeitos Furacão e Norma ao longo dos passos de treino no Experimento I.

Em outras palavras, quanto maior a quantidade de discriminações simples aprendidas, menor o tempo necessário para que o organismo obtenha o critério de aprendizagem exigido para uma nova discriminação simples.

Os dados de Furacão e Norma não apresentam indícios de ocorrência de learning set. Apesar das sucessivas reversões realizadas, principalmente no caso de Furacão, a quantidade de sessões necessárias para a obtenção do critério variou em ordem assistemática, sem apresentar uma tendência decrescente como seria característico da ocorrência de learning set. É possível sugerir que a quantidade de reversões realizadas foi insuficiente para que os sujeitos pudessem apresentar qualquer indício de learning set; porém, resultados similares que sugerem a dificuldade em evidenciar a ocorrência desse padrão comportamental em cães também foram descritas em outros experimentos que ensinaram discriminações simples com reversões sucessivas (Dahás, Brasiliense, Barros, Costa, \& Souza, 2010; Domeniconi, Bortoloti, Antoniazzi, \& Mendes, 2008; Montagnoli, 2012). Em virtude da ausência de learning set e da grande quantidade de sessões realizadas com cada um dos sujeitos, optou-se pela descontinuação do procedimento utilizado no Experimento I e pela realização de um segundo experimento utilizando outro procedimento de treino.

\section{EXPERIMENTO II}

O segundo experimento teve por objetivo adaptar o protocolo de ensino de discriminações condicionais proposto por Dube (1996) para a coleta com cães. Resumidamente, Dube argumenta que um desempenho adequado em discriminações condicionais pode ser obtido utilizando um treino iniciado por discriminações simples. O desempenho adequado nas discriminações simples poderia ser a linha de base inicial para a inserção de um estímulo modelo em cada tentativa e, assim, gerar um desempenho controlado por discriminações condicionais.

Um aspecto observado a partir da análise de dados do Experimento I refere-se à grande quantidade de sessões necessárias para a obtenção do critério. Furacão, por exemplo, necessitou de 106 sessões (ou, aproximadamente 5 meses) para atingir o critério de aprendizagem exigidos no experimento anterior. Seria necessário, portanto, desenvolver procedimentos de ensino que pudessem gerar os padrões comportamentais alvo com quantidade menor de treino (Barros, Galvão, \& McIlvane, 2003; Galvão, Barros, Rocha, Mendonça, \& Goulart, 2002). Nesse caso, a utilização de estímulos distratores poderia auxiliar o processo de aquisição das discriminações simples. O raciocínio é consideravelmente simples: ao utilizar um único par de estímulos que se alternam em suas funções de correto e incorreto (ou $\mathrm{S}+\mathrm{e}$ $\mathrm{S}$-), exige-se dos sujeitos não apenas a aquisição de uma 
nova discriminação simples, mas a reversão de uma discriminação simples anteriormente aprendida. Por exemplo, em um treino que utilize apenas os estímulos A1 e A2, no qual a escolha de A1 tenha sido previamente reforçada, uma reversão exige não só que o sujeito passe a escolher o estímulo de A2, mas também que as escolhas de A1 sejam extintas.

Ao utilizar estímulos distratores, não há necessidade de extinguir escolhas aprendidas anteriormente. Pode-se, por exemplo, ensinar uma discriminação simples com os estímulos A1 e B1, sendo reforçada a escolha de A1. Uma vez atingido o critério de aprendizagem para essa tarefa, o par de estímulos A1 e B1 é substituído pelo par A2 e B2, sendo agora reforçada a escolha de B2. Assim, a escolha de A2 não exige a extinção da escolha de A1 e, por esse motivo, a probabilidade de erros é diminuída. Nesse contexto, os estímulos B1 e B2 são chamados de distatores porque eles possuem a função de compor as tentativas, mas esses estímulos nunca exercem a função de $\mathrm{S}+$.

Assim sendo, ainda utilizando o mesmo aparato automatizado, o Experimento II teve por objetivo estabelecer discriminações condicionais em cães adaptando o protocolo de ensino proposto por Dube (1996) e utilizando estímulos de comparação distratores para diminuir a ocorrência de erros e, consequentemente, a quantidade necessária de treino. $\mathrm{O}$ procedimento de treino blocado (Saunders \& Spradlin, 1990, 1993), utilizado no Experimento I, foi novamente utilizado no Experimento II para o ensino das discriminações simples.

\section{Sujeitos \\ MÉTODO}

Dois sujeitos foram submetidos ao Experimento II. Norma, que fora anteriormente submetida ao Experimento I, e Pipoca, que era experimentalmente ingênua (ver Tabela 1).

\section{Local, situação experimental e equipamento}

Idêntico ao descrito para o Experimento I.

\section{Procedimento}

Antes de iniciar o procedimento, Pipoca foi submetida ao treino de modelagem. Norma, por sua vez, iniciou os passos de ensino propostos no Experimento II uma semana após encerrar sua participação no Experimento I.

O Experimento II era composto por oito passos. No Passo 1, cada sessão tinha 72 tentativas. As tentativas eram iniciadas com a apresentação simultânea dos estímulos nas posições laterais da tela, sendo que a posição de apresentação dos estímulos era balanceada ao longo das tentativas. A escolha de $\mathrm{C} 1$ produzia a apresentação do padrão sonoro agudo e, simultaneamente, a entrega de uma unidade de ração canina. Em seguida, tinha início um IET de 2s. Durante o IET, os estímulos eram retirados da tela e eventuais respostas emitidas pelos sujeitos não produziam alterações ambientais programadas.
A escolha de C2 produzia, simultaneamente, a apresentação do padrão sonoro grave, o escurecimento da tela (time out) por $3 \mathrm{~s}$ e o IET. A escolha do estímulo de comparação incorreto ocasionava a reapresentação da mesma tentativa e, caso o sujeito cometesse três erros consecutivos em uma mesma tentativa, somente o estímulo de comparação correto era apresentado na próxima oportunidade de resposta (Procedimento de Correção A). O critério de aprendizagem nesse passo foi estabelecido em porcentagens iguais ou superiores a $80 \%$ de acertos em duas sessões consecutivas.

O Passo 2 era idêntico ao que foi descrito para o Passo 1 no que se refere ao local de apresentação dos estímulos, ao número de tentativas, às consequências diferenciais para acertos ou erros e ao critério de aprendizagem. A única diferença estava no par de estímulos apresentados, D1, cuja escolha era considerada correta, e D2, cuja escolha era considerada incorreta.

O Passo 3 era dividido em dois blocos, o primeiro bloco de 36 tentativas apresentava o par de estímulos $\mathrm{C} 1 \mathrm{e}$ C2; o segundo bloco de 36 tentativas apresentava o par de estímulos D1 e D2. No Passo 4, eram apresentados de forma alternada para os sujeitos três blocos de 12 tentativas com os estímulos $\mathrm{C} 1$ e $\mathrm{C} 2$ e outros três blocos de 12 tentativas com os estímulos D1 e D2. No Passo 5, de forma alternada, eram apresentados seis blocos de seis tentativas os estímulos do conjunto $\mathrm{C}$ e outros seis blocos de seis tentativas com os estímulos do conjunto D. Por fim, o Passo 6 alternava a apresentação de 18 blocos de duas tentativas com os estímulos do Conjunto $\mathrm{C}$ e outros 18 blocos de duas tentativas com os estímulos do Conjunto D. Os demais aspectos do procedimento permaneciam iguais.

No Passo 7, os estímulos que deveriam exercer a função de modelo eram introduzidos na tarefa e as tentativas eram iniciadas com sua apresentação na posição central da tela. Focinhar esse estímulo produzia a retirada do mesmo e a apresentação imediata de dois estímulos de comparação nas posições laterais da tela, sendo um deles fisicamente idêntico ao modelo e cuja escolha era consequenciada com ração. As sessões apresentavam 36 tentativas, sendo 18 delas com o estímulo $\mathrm{C} 1$ como modelo (e os estímulos $\mathrm{C} 1$ e C2 como comparações) e 18 com o estimulo D1 como modelo (tendo os estímulos D1 e D2 como comparações). A ordem de apresentação dos estímulos modelo, e suas respectivas comparações, eram aleatórias e as sessões repetidas até que o animal acertasse $80 \%$ das tentativas em três de quatro sessões consecutivas.

O Passo 8 também apresentava 36 tentativas, mas as configurações das tentativas poderiam ser de 4 tipos: em 15 tentativas, o estímulo modelo era $\mathrm{C} 1$ e os estímulos de comparação eram $\mathrm{C} 1$ e C2; em 15 tentativas, o estimulo modelo era D1 e os comparações D1 e D2; em três tentativas o modelo $\mathrm{C} 1$ era apresentado com os comparações C1 e D1; e em três tentativas o modelo D1 era apresentado com os comparações C1 e D1. A ordem de apresentação destas tentativas era aleatória. Foram realizadas seis e sete sessões do Passo 8 com Norma e Pipoca, respectivamente. Vale ressaltar que o 
Procedimento de Correção A continuava em vigor nos passos 7 e 8 .

Dois aspectos merecem atenção especial nesse procedimento. $\mathrm{O}$ primeiro refere-se ao fato de que os estímulos C2 e D2, apesar de serem apresentados como comparações, nunca tiveram suas escolhas reforçadas e, por esse motivo, são chamados de distratores. Assim, os sujeitos expostos a esse procedimento não tiveram que reverter discriminações simples aprendidas anteriormente, mas, ao invés disso, aprender duas discriminações simples independentes.

O segundo aspecto refere-se ao desempenho diante das seis tentativas em que os estímulos de comparação C e D eram apresentados juntos. Nessa configuração, apesar de o responder já ter sido reforçado diante de qualquer um dos estímulos de comparação, apenas o responder sob controle do estímulo modelo garantiria o acesso ao reforçador. Desta forma, essas tentativas poderiam ser consideradas como sondas que evidenciariam discriminação condicional em cães, caso o responder fosse correto.

\section{RESULTADOS E DISCUSSÃO}

A Figura 3 apresenta os desempenhos de Norma e Pipoca nos oito passos de ensino. As porcentagens de acertos obtidas por Norma e Pipoca estão apresentadas nos painéis superior e inferior, respectivamente. Barras pretas e cinzas representam, respectivamente, as porcentagens de acertos em tentativas em que eram ensinadas as discriminações simples C1/C2 e D1/D2. Círculos pretos e cinzas representam, respectivamente, as porcentagens de acertos nas tentativas em que C1 e D1 eram apresentados como modelos. Triângulos pretos representam as porcentagens de acertos nas tentativas em que o estímulo C1 era apresentado como modelo e os estímulos C1 e D1 eram apresentados como comparações; triângulos cinzas representam as porcentagens de acertos nas tentativas em que o estímulo D1 era apresentado como modelo e os mesmos estímulos C1 e D1 eram apresentados como comparações. A linha tracejada horizontal identifica o critério de aprendizagem. Abaixo do eixo X é apresentado o número de sessões realizado em cada passo. Para o cálculo das porcentagens de acertos, as tentativas que necessitaram de correção foram consideradas como erros.

Ambos os sujeitos apresentaram padrões de aquisição similares. Os sujeitos iniciaram o treino da primeira discriminação simples simultânea (P1) com porcentagens próximas aos $50 \%$ de acertos. Norma atingiu o critério de aprendizagem em 20 sessões e pipoca em nove sessões

Ao comparar a quantidade de sessões necessárias para concluir os passos 1 e 2, é possível observar indícios de learning set no desempenho dos dois sujeitos. Norma atingiu o critério de aprendizagem no P2 em um número menor de sessões quando comparado ao P1 (reduziu de 20 para seis sessões). Pipoca também concluiu o P2 em número menor de sessões, embora a diferença nos valores não tenha sido tão expressiva (de nove para cinco sessões). Além disso, na primeira sessão com o segundo par de estímulos (primeira sessão de P2), as porcentagens de acertos foram superiores a 70\%, fato que também sugere ocorrência de learning set.

Uma vez aprendidas as discriminações simples nos Passos 1 e 2, o fato delas serem apresentadas alternadamente numa mesma sessão pareceu não interferir no desempenho dos sujeitos. Nos Passos 3, 4, 5 e 6, para ambos os sujeitos, as porcentagens de acertos ficaram próximas ou acima dos $80 \%$.

No Passo 7, a inserção do estímulo modelo pareceu não afetar o desempenho dos sujeitos, pois as porcentagens de acertos permaneceram elevadas desde a primeira sessão, com o critério de aprendizagem sendo atingido na quantidade mínima de sessões previstas (quatro sessões). Esses resultados sugerem que o estímulo modelo poderia não estar exercendo o controle planejado no responder dos animais, uma vez que a variável ausência (P6) ou presença (P7) do modelo não causou qualquer alteração no desempenho observado.

Em P8, nas tentativas em que a escolha ocorria entre dois estímulos de comparação cujas escolhas já haviam sido reforçadas em passos anteriores (C1 e D1), o desempenho deteriorou consideravelmente (Triângulos pretos e cinzas). Norma atingiu porcentagens de acertos que variaram entre aproximadamente $33 \%$ nas duas primeiras sessões e $50 \%$ na sexta e última sessão. Pipoca, por sua vez, atingiu aproximadamente $66 \%$ de acertos na primeira sessão de $\mathrm{P} 8$, porém esse desempenho não foi mantido nas demais sessões, com porcentagens variando entre $33 \%$ e $50 \%$ de acertos. Ainda assim, os cães continuaram a obter porcentagens de acertos próximos a $80 \%$ nas tentativas que apresentavam os estímulos distratores.

Provavelmente, os sujeitos aprenderam a rastrear os estímulos com função de $\mathrm{S}+$ ou então a excluir o $\mathrm{S}$ - nos passos que ensinavam as discriminações simples. Esse mesmo padrão de rastreamento do $\mathrm{S}+$ e/ou do $\mathrm{S}$ - também se mostrou eficaz para atingir o critério de aprendizagem no Passo 7. Contudo, quando os estímulos C1 e D1 passaram a ser apresentados simultaneamente como estímulos de comparação, os sujeitos tinham a mesma história de reforço na presença de cada um deles. Nesse sentido, a tarefa se tornou ambígua e uma distribuição igualitária das respostas entre ambos os estímulos seria um dado compatível com a história experimental. Os resultados obtidos parecem confirmar essa hipótese.

\section{DISCUSSÃO GERAL}

Em ambos os experimentos, os procedimentos empregados foram ineficazes em estabelecer discriminações controladas por relações condicionais. Apenas evidências do estabelecimento de discriminações simples foram providas pelos resultados obtidos. No Experimento I, a grande quantidade de sessões requeridas para a obtenção dos critérios de aprendizagem nas Condições 1 e 2 e a falta de evidências que indicassem o learning set impediram que ambas as relações modelocomparação (i.e., A1-A1 e A2-A2) fossem apresentadas em uma mesma sessão, portanto, um comportamento controlado por relações condicionais nem chegou a ser requerido. 

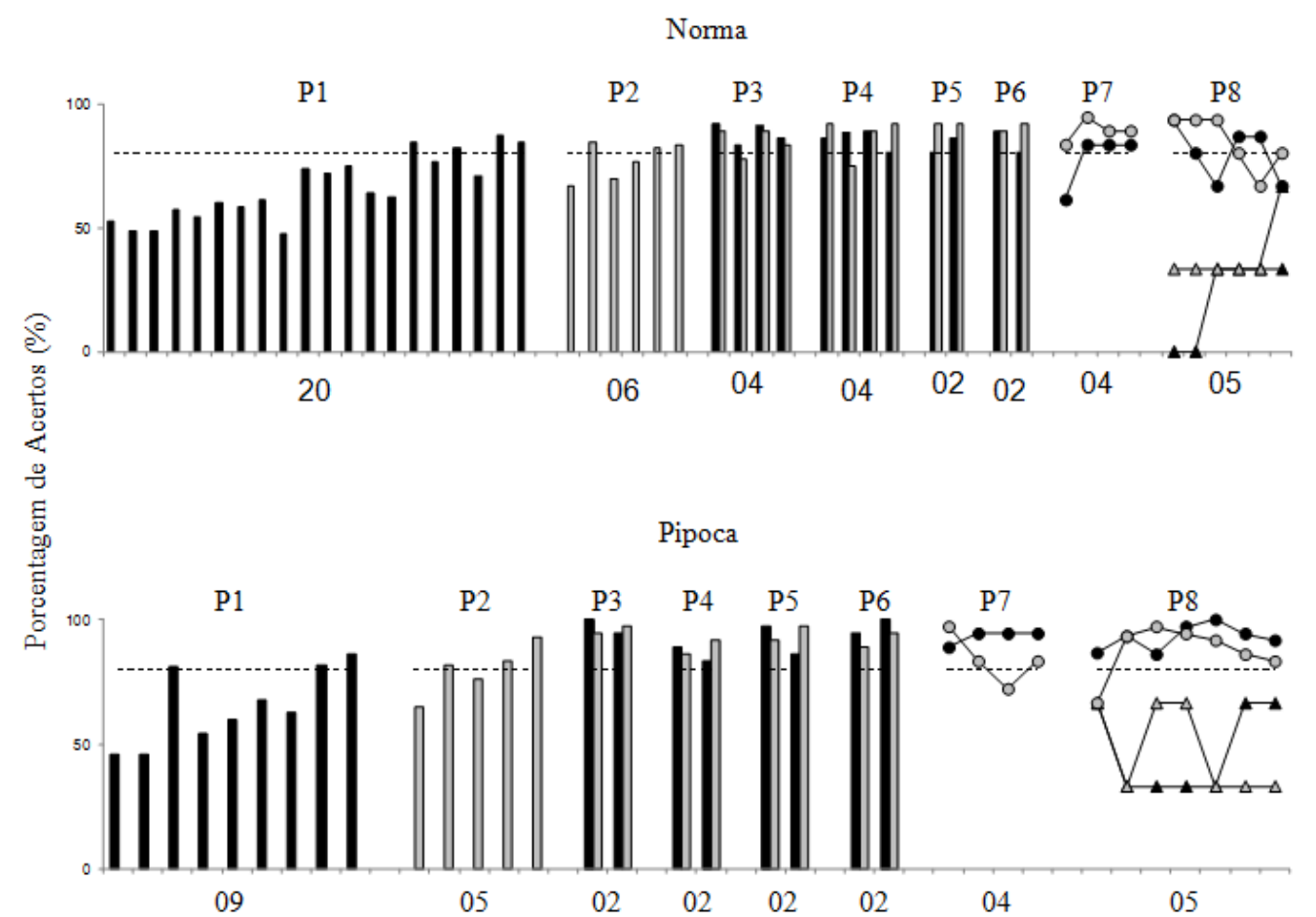

Quantidade de Sessões Realizadas em cada Passo

Figura 3. Porcentagem de acertos obtida por Norma e Pipoca ao longo de todos dos passos de treino no Experimento II.

No Experimento II, ao utilizar estímulos distratores, o procedimento também permitiu o estabelecimento do controle apenas pelo estímulo que sinalizava a obtenção do reforço (discriminação simples), sem exigir que uma situação de condicionalidade fosse exigida. Apesar de ambas as estratégias serem utilizadas com relativo sucesso em experimentos conduzidos com outros sujeitos não humanos (Barros et al., 2003; Galvão et al., 2002; Goulart et al., 2003), os mesmos resultados não foram encontrados com cães. Ao menos no que concerne a esses sujeitos, as variáveis que influenciam a aquisição de discriminação condicional não foram completamente examinadas e poderiam ser alvo de investigações futuras.

Outro aspecto do procedimento que pareceu influenciar nos resultados encontrados foi a utilização de blocos de tentativas apresentando a mesma relação condicional (treino blocado - Saunders \& Spradlin, 1990, 1993). Ao considerar que as tentativas são sequencialmente repetidas, inexiste uma contingência que exija a alternância das respostas e o modelo torna-se um estímulo irrelevante na tarefa. Futuros experimentos poderiam utilizar procedimentos que sejam iniciados por tentativas dispostas em sequência aleatória.

Em comparação com estudos que observaram desempenho compatível com discriminação condicional em cães, dois aspectos devem ser considerados. Primeiro, o experimento conduzido por Kaminski et al. (2009) utilizou estímulos tridimensionais enquanto os estímulos ora utilizados eram bidimensionais. A capacidade de cães em discriminar e reconhecer estímulos bidimensionais foi anteriormente comprovada em diversos estudos (p. ex., Adachi et al., 2007; Aust et al., 2008; Range et al., 2008); entretanto, é possível sugerir que estímulos tridimensionais possuem grau superior de discriminabilidade, o que poderia facilitar o desempenho dos cães nas tarefas de discriminação condicional.

O segundo aspecto refere-se ao fato de a tarefa utilizada por Kaminski et al. (2009) ser consideravelmente familiar ao cão. É comum, por exemplo, brincadeiras envolvendo comandos verbais tais como "dá-me o osso" ou "onde está a bola", diante dos quais os cães são requisitados a buscar objetos específicos. No caso da tarefa experimental treinada nos experimentos aqui descritos, é possível verificar que não existem situações cotidianas nos quais os cães são treinados a discriminar condicionalmente objetos similares. Em outras palavras, a relação condicional entre dois estímulos visuais não encontra interações similares em atividades cotidianas que envolvam cães e seus respectivos donos. Talvez, em futuras pesquisas, deva-se considerar a possibilidade de trabalhar em contextos experimentais menos arbitrários ou com maior validade ecológica, a fim de garantir um desempenho mais próximo do que pode ser observado no ambiente natural desses animais.

A análise dos resultados dos experimentos não indicou o estabelecimento de relações condicionais nos sujeitos. Apesar disso, é possível identificar aspectos 
positivos decorrentes de sua realização. Como primeiro resultado relevante pode-se elencar o desenvolvimento de um aparato e de uma configuração experimental adequada para a condução de pesquisas sobre discriminações com cães, sujeitos experimentais que ainda não possuem uma história experimental longa, quando comparados aos demais sujeitos não humanos utilizados em pesquisas nessa área (e.g., pombos, ratos, macacos - Udell \& Wynne, 2008). Vale ressaltar que a utilização de um software para apresentação dos estímulos antecedentes e consequentes no ensino de discriminações condicionais resolve parte dos problemas encontrados em estudos conduzidos com cães, nos quais a alta sensibilidade desses animais para detectar e reagir a dicas sociais humanas não é controlada (Montagnoli, 2012).

$\mathrm{O}$ desenvolvimento de novos aparatos e tecnologias para o estudo de processos comportamentais e avaliação das capacidades cognitivas em cães parece atrair cada vez mais interessados. Williams, Mills e Guo (2011), por exemplo, aperfeiçoaram um sistema de rastreamento do olhar para ser utilizado com cães. Segundo os autores, o interesse por esses sujeitos experimentais está no fato de sua reconhecida expertise em reconhecer e se adaptar a dicas sociais. Essa quantidade substancial de experimentos com cães, acompanhado pelo desenvolvimento de aparatos e tecnologias específicas para esses sujeitos, parecem indicar que o estudo do comportamento simbólico com modelos animais caninos deverá produzir bons resultados no futuro.

Em segundo lugar, os procedimentos empregados conseguiram instalar nos sujeitos uma cadeia comportamental consideravelmente extensa que consistia em (i) permanecer diante da tela durante toda a sessão, sem a necessidade de utilizar equipamentos para restringir seus movimentos, (ii) rastrear a tela à procura de um estímulo visual que poderia ser apresentado em diferentes lugares, (iii) focinhar a tela nos lugares em que os estímulos eram apresentados e (iv) obter ração diante da escolha de estímulos específicos. O conhecimento decorrente da realização desses experimentos certamente será útil em outras pesquisas que requeiram estes repertórios como requisitos para outros comportamentos. Tal avanço poderia originar vários procedimentos experimentais para o estudo não somente do comportamento simbólico, mas também de processos como memória e percepção.

\section{REFERÊNCIAS}

Adachi, I., Kuwahata, H., \& Fujita, K. (2007). Dogs recall their owner's face upon hearing the owner's voice. Animal Cognition, 10, 17-21.

Barros, R. S., Galvão, O. F., \& McIlvane, W. J. (2003). Search for relational learning capacity in Cebus apella: A programmed "educational" approach. Em S. Soraci$\mathrm{Jr}$ e K. Murata-Soraci (Orgs.). Visual information processing. Westport, CT: Praeger.

Callahan, H., Ikeda-Douglas, C., Head, E., Cotman, C. W., \& Milgram, N. W. (2000). Development of a protocol for studying object recognition memory in the dog.
Progress in Neuro-Psychopharmacology \& Biological Psychiatry, 24, 693-707.

Capaldi, E. J., \& Senko, M. G. (1961). Effect of trials-perproblem on successive discrimination reversal learning. Psychological Reports, 8, 227-232.

Dahás, L. J. S., Brasiliense, I. C. S., Barros, R. S., Costa, T. D., \& Souza, C. B. A. (2010). Formação de classes funcionais em cães domésticos (Canis familiaris): Uma abordagem da aquisição de comportamento présimbólico. Acta Comportamentalia, 18, 317-346.

Dixon, L. S. (1977). The nature of control by spoken words over visual stimulus selection. Journal of the Experimental Analysis of Behavior, 27, 433-442.

Domeniconi, C., Bortoloti, R., Antoniazzi, L. C. K., \& Mendes, T. E. N. (2008). Treinos de discriminações simples e formação de classes funcionais de estímulos por cães. Interação em Psicologia, 12, 235-243.

Dorey, N. R., Udell, M. A. R., \& Wynne, C. D. L. (2009). Breed differences in dogs sensitivity to human points: A meta analysis. Behavioral Processes, 81, 409-415.

Dube, W. V. (1996). Teaching discrimination skills to person with mental retardation. Em C. Goyos, M. A. Almeida \& D. G. de Souza (Orgs.), Temas em Educação Especial (Pp. 73-96). São Carlos: EDUFSCar.

Fiset, S., Landry, F., \& Ouellette, M. (2006). Egocentric search for disappearing objects in domestic dogs: Evidence for a geometric hypothesis of direction. Animal Cognition, 9, 1-12.

Fiset, S., \& LeBlanc, V. (2007). Invisible displacement understanding in domestic dogs (Canis familiaris): The role of visual cues in search behavior. Animal Cognition, 10, 211-224.

Galvão, O. F., Barros, R. S., Rocha, A. C., Mendonça, M. B., \& Goulart, P. R. K. (2002). Escola Experimental de Primatas. Estudos de Psicologia, 7, 361-370.

Gazit, I., Goldblatt, A., \& Terkel, J. (2005). The role of context specificity in learning: The effects of training context on explosives detections in dogs. Animal Cognition, 8, 143-150.

Goulart, P. R. K., Galvão, O. F., \& Barros, R. S. (2003). Busca de formação de classes de estímulos via procedimento de reversões repetidas de discriminações simples em macaco-prego (Cebus apella). Interação, 7,109-119.

Hare, B., Call, J., \& Tomasello, M. (1998). Communication of food location between human and dog (Canis familiaris). Evolution of Communication, 2, 137-159.

Harlow, H. F. (1949). The formation of learning sets. Psychological Review, 56, 51-65.

Heberlein, M., \& Turner, D. C. (2009). Dogs, Canis familiaris, find hidden food by observing and 
interacting with a conspecific. Animal Behaviour, 78, 385-391.

Ittyerah, M., \& Gaunet, F. (2009). The response of guide dogs and pet dogs (Canis familiaris) to cues of human referential communication (pointing and gaze). Animal Cognition, 12, 257-265.

Kaminski, J., Call, J., \& Fischer, J. (2004). Word learning in a domestic dog: Evidence for "fasting mapping". Science, 304, 1682-1683.

Kaminski, J., Tempelmann, S., Call, J., \& Tomasello, M. (2009). Domestic dogs comprehend human communication with iconic signs. Developmental Science, 12, 831-837.

Milgram, N. W., Head, E., Zicker, S. C., Ikeda-Douglas, C., Murphey, H., Muggenberg, B. A., Siwak, C. T., Tapp, P. D., Lowry, S. R., \& Cotman, C. W. (2004). Long-term treatment with antioxidants and a program of behavioral enrichment reduces age-dependent impairment in discrimination and reversal learning in beagle dogs. Experimental Gerontology, 39, 753-765.

Miller, H. C., Rayburn-Reeves, R., \& Zentall, T. R. (2009). What do dogs know about hidden objects? Behavioural Processes, 81, 439-446.

Montagnoli, T. A. S. (2012). Um procedimento para investigar aprendizagem discriminativa e formação de classes funcionais em cães. Dissertação de Mestrado. São Carlos: Universidade Federal de São Carlos.

Pilley, J. W., \& Reid, A. K. (2011). Border collie comprehends object names as verbal referents. Behavioural Processes, 86, 184-195.

Saunders, K. J., \& Spradlin, J. E. (1990). Conditional discrimination in mentally retarded adults: The development of generalized skills. Journal of the Experimental Analysis of Behavior, 54, 239-250.

Saunders, K. J., \& Spradlin, J. E. (1993). Conditional discrimination in mentally retarded subjects: Programming acquisition and learning set. Journal of the Experimental Analysis of Behavior, 60, 571-585.

Osthaus, B., Lea, S. E. G., \& Slater, A. M. (2005). Dogs (Canis lupus familiaris) fail to show understanding of means-end connections in a string-pulling task. Animal Cognition, 8, 37-47.
Range, F., Aust, U., Steurer, M., \& Huber, L. (2008). Visual categorization of natural stimuli by domestic dogs. Animal Cognition, 11, 339-347.

Rossi, A. P., \& Ades, C. (2008). A dog at the keyboard: Using arbitrary signs to communicate requests. Animal Cognition, 11, 329-338.

Sidman, M., \& Tailby, W. (1982). Conditional discrimination vs. matching to sample: An expansion of the testing paradigm. Journal of the Experimental Analysis of Behavior, 37, 5-22.

Tapp, P. D., Siwak, C. T., Estrada, J., Head, E., Muggenburg, B. A., Cotman, C. W., \& Milgram, N. W. (2011). Size and reversal learning in the beagle dog as a measure of executive function and inhibitory control in aging. Learning \& Memory, 10, 64-73.

Topál, J., Byrne, R. W., Miklósi, A., \& Csányi, V. (2006). Reproduction human actions and action sequences: "Do as I do" in a dog. Animal Cognition, 9, 355-367.

Williams, F. J., Mills, D. S., \& Guo, K. (2011). Development of a head-mounted, eye-tracking system for dogs. Journal of Neuroscience Methods, 194, 259265.

Udell, M. A. R., \& Wynne, C. D. L. (2008). A review of domestic dogs' (Canis familiaris) human-like behaviors: Or why behavior analysts should stop worrying and love their dogs. Journal of the Experimental Analysis of Behavior, 89, 247-261.

Vaughan, W., Jr. (1988). Formation of equivalence sets in pigeons. Journal of Experimental Psychology: Animal Behavior Processes, 14, 36-42.

Velasco, S. M., \& Picorone, A. M., (2008). Stimulus control 1002. Laboratório de Análise do Comportamento. São Paulo: IPUSP. Software desenvolvido para a condução de pesquisas com não humanos.

Submetido em 10/04/2014. Aceito em 22/06/2015. Publicado em 01/02/2016 